\title{
Endoscopic Ultrasound-Guided Local Therapy for Pancreatic Neoplasms
}

\author{
Jun Seong Hwang ${ }^{1}$, Hyun Don J00 ${ }^{2}$ and Tae Jun Song ${ }^{2}$ \\ ${ }^{1}$ Department of Internal Medicine, Haeundae Paik Hospital, Inje University College of Medicine, Busan, ${ }^{2}$ Department of \\ Gastroenterology, Asan Medical Center, University of Ulsan College of Medicine, Seoul, Korea
}

Surgical resection is considered the only treatment option for pancreatic cancer and other pancreatic neoplasms with malignant potential, such as neuroendocrine tumors, mucinous cystic neoplasms, and intraductal papillary mucinous neoplasms. However, only $10 \%-20 \%$ of all patients with pancreatic cancer present with resectable forms of the disease as the symptoms are rarely manifested during the early stages, and the disease tends to progress rapidly. Furthermore, pancreatic surgery is associated with high rates of morbidity and mortality. The development of linear-array endoscopic ultrasound (EUS) techniques has increased the indications of EUS-guided local therapy for pancreatic neoplasms. We assessed the studies that investigated various treatment modalities, such as fine-needle injection, radiofrequency ablation, irreversible electroporation, and radiotherapy, under EUS guidance to better understand the usefulness of these techniques with respect to the efficacy and associated complications. Clin Endosc 2020;53:535-540

Key Words: Endoscopic ultrasound; Local treatment; Pancreatic cancer; Pancreatic neoplasms

\section{INTRODUCTION}

There are several types of primary pancreatic neoplasms, such as pancreatic cancer, neuroendocrine tumors, and cystic neoplasms. The prognosis of patients with pancreatic ductal adenocarcinomas is the poorest; its prevalence has gradually increased, and pancreatic ductal adenocarcinoma was the ninth most common cancer in Korea as of 2016. ${ }^{1}$ Although surgical resection is the only therapeutic option for improving long-term survival rates, only $10 \%-20 \%$ of all patients with pancreatic cancer can undergo surgery at the time of diagnosis. Owing to the absence of characteristic symptoms during the early stages of the disease, fast growth rate of the

Received: July 7, 2020 Revised: September 7, 2020

Accepted: September 17, 2020

Correspondence: Tae Jun Song

Department of Gastroenterology, Asan Medical Center, University of Ulsan College of Medicine, 88 Olympic-ro 43-gil, Songpa-gu, Seoul 05505, Korea Tel: +82-2-3010-3914, Fax: +82-2-476-0824, E-mail: drsong@amc.seoul.kr ORCID: https://orcid.org/0000-0002-6156-8746

(c) This is an Open Access article distributed under the terms of the Creative Commons Attribution Non-Commercial License (http://creativecommons.org/ licenses/by-nc/3.0) which permits unrestricted non-commercial use, distribution, and reproduction in any medium, provided the original work is properly cited. tumor, and easy infiltration and metastasis to the surrounding tissues and lymph nodes, surgery is not performed at the time of diagnosis for most patients. ${ }^{2}$ The standard treatment for unresectable pancreatic cancers has not been established to date. Although chemotherapy and radiotherapy with anticancer drugs have been the main alternatives, most patients show limited response to these treatments. ${ }^{3}$ Pancreatic cystic neoplasms include intraductal papillary mucinous neoplasms, mucinous cystic neoplasms, serous cystic adenomas, and solid pseudopapillary tumors. Some of these can become malignant; therefore, surgical treatment is recommended when the risk of malignancy is high. ${ }^{4}$ However, pancreatic surgery, especially pancreaticoduodenectomy and pylorus-preserving pancreaticoduodenectomy, is associated with high rates of morbidity and mortality. ${ }^{5}$ The recent development of endoscopic ultrasound (EUS) equipment and techniques has led to an increase in the application of EUS-guided local therapy for pancreatic neoplasms. Local endoscopic treatment for unresectable pancreatic cancer is effective in reducing the tumor size and alleviating pain. ${ }^{6}$ This article provides an overview of the types, effects, and complications of EUS-guided local therapy, such as EUS-guided fine-needle injection (EUS-FNI) of chemotherapeutic agents or viral vectors (virotherapy), radiof- 
Table 1. Local Endoscopic Treatment for Pancreatic Neoplasm

Injection therapy

(1) Chemotherapeutic agents

(2) Viral and immunologic vectors

Ablation therapy

(1) Radiofrequency ablation

(2) Cryotherapy

Radiotherapy

(1) Fiducial marker insertion

(2) Brachytherapy

Drug delivery stents

requency ablation (RFA), irreversible electroporation (IRE), and fiducial marker insertion, for pancreatic neoplasms. (Table 1).

\section{ENDOSCOPIC ULTRASOUND-GUIDED FINE-NEEDLE INJECTION}

Currently, EUS-FNI is most commonly used for treating pancreatic cysts. However, the application of EUS-FNI for solid tumors, particularly pancreatic cancer, is limited in the clinical setting. The effect of chemotherapy on pancreatic cancer is inferior to that on other carcinomas; the drug transport rate to the tumor is low owing to the presence of fewer blood vessels and intense connective tissue proliferation (desmoplasia) in the tumor microenvironment. ${ }^{7}$ Local injection of anticancer agents into tumors via EUS-FNI is expected to reduce the systemic adverse events associated with chemotherapy and increase the drug transport rate to tumors. Experimental results have been reported for several injectable formulations. However, previous studies have not shown comprehensible effects, such as prolonged survival.

\section{Chemotherapeutic agents}

\section{Gemcitabine}

Levy et al. ${ }^{8}$ injected gemcitabine in 36 patients (34 unresectable pancreatic cancer, 1 poor operative candidate, and 1 refusal of resection) under EUS guidance. After administering $40 \mathrm{mg} / \mathrm{mL}$ gemcitabine via EUS-FNI, the 6-month and 1-year survival rates in the patient group were $76 \%$ and $46 \%$, respectively. The cancer was down-staged to a resectable lesion in three patients, all of whom underwent R0 resection.

\section{OncoGel}

In 2007, Matthes et al. ${ }^{9}$ reported experimental results for
EUS-guided injection of a paclitaxel formulation (OncoGel; MacroMed Inc., Salt Lake City, UT, USA) into the pig pancreas. In the pancreas of eight pigs, 1-4 mL of OncoGel was injected under EUS guidance. In all cases, the experiment was completed without complications, such as pancreatitis or intraperitoneal infection. Paclitaxel concentration was maintained within the therapeutic range in the pancreatic tissue 14 days after drug injection; however, the appropriate drug concentration was not maintained at a distance of $30-50 \mathrm{~mm}$ or more from the injection site. In addition, when injecting an anticancer agent using EUS-FNI, accurate needle puncture and correct volume of injection into the tumor are difficult to achieve owing to the high-density fibroses accompanying pancreatic cancers.

In summary, although intratumoral anticancer agent injection via EUS-FNI was expected to effectively induce tumor cell death, there was no apparent improvement in the survival rate of pancreatic cancer patients. Therefore, further research is required with respect to the therapeutic applications.

\section{Viral and biological vectors}

\section{Viral therapy}

Anticancer therapies for unresectable pancreatic cancers using viruses have been studied, with a focus on the selectivity of certain genetically engineered viruses for the tumor and their ability to induce cytolysis in tumor cells. These viruses target only the genes of malignant cells and are genetically engineered to prevent damage to normal cells. ${ }^{10}$ For unresectable pancreatic cancer, EUS-guided anticancer therapies using adenovirus and herpes virus have been devised, but most of them have not shown significant results. ${ }^{11,12}$

\section{Immunotherapy}

In addition to viral therapy, studies have been conducted on the intratumoral administration of immunotherapeutic agents under EUS guidance to improve the local immune response in patients with pancreatic cancer.

One of the first candidates is the cytoimplant. Chang et al. ${ }^{13}$ reported the results of a phase I clinical trial in which eight patients with advanced pancreatic cancer were injected with an allogeneic mixed lymphocyte culture under EUS guidance. Cell implants were generated by co-culturing mononuclear cells in the peripheral blood of healthy donors and patients. The cytoimplant was expected to release cytokines and activate immune cells; however, only two patients showed partial response to treatment, and the median survival rate was 13.2 months.

The second candidate is dendritic cell injection under EUS guidance. Dendritic cell implantation was first reported by 
Nonogaki et al. ${ }^{14}$ in 2007. Since then, several small-scale studies have been conducted, but none have shown meaningful results, such as prolonged survival.

The third option is TNFerade injection under EUS guidance. TNFerade is a replication-deficient adenovirus vector carrying the human tumor necrosis factor-alpha (TNF-alpha) gene regulated by a radiation-inducible promoter (early growth response-1). Hecht et al. ${ }^{15}$ published the results of phase I/II clinical trials combining TNFerade injection with chemoradiotherapy in 50 patients with pancreatic cancer. TNFerade was injected via EUS-FNI or percutaneously into unresectable pancreatic cancers, and patients received a radiotherapy dose of $50.4 \mathrm{~Gy}$ and intravenous administration of 5-FU $\left(200 \mathrm{mg} / \mathrm{m}^{2}\right)$ every day for 5.5 weeks. One patient showed complete treatment response, three showed partial treatment response, and 12 showed stable disease persistence. A total of seven patients underwent surgical resection, of which six underwent R0 resection. Of these, three patients survived for more than 24 months.

\section{LOCAL ABLATIVE THERAPY}

Local ablation of tumors using heat or lasers is widely used for treating various solid cancers. Interest in local treatment for unresectable pancreatic cancer has also increased with the development of endoscopic techniques, equipment, and imaging techniques. Various methods of applicable local treatment have been developed with the expectation that they will result in a reduction in the tumor size and downstaging of the tumor. Among the various local treatments, RFA and IRE represent thermal and non-thermal ablative techniques, respectively, and their effectiveness and safety have been well studied.

\section{Endoscopic ultrasound-guided radiofrequency ablation}

EUS-RFA is a local treatment that uses heat generated by the agitation of ions in cells to induce cell death and coagulation necrosis. Ion agitation is achieved by applying a high-frequency alternating current of approximately $500 \mathrm{kHz} .{ }^{16} \mathrm{In}$ addition to the thermal ablation effect, RFA appears to play a role in the secondary anticancer immune response by inducing activation of tumor-specific $\mathrm{T}$ lymphocytes and expression of heat shock protein $70 .{ }^{17}$ RFA has been widely used for the treatment of solid cancers in organs such as the liver, lungs, and kidneys. However, it is not often used in the treatment of pancreatic tumors due to the risk of pancreatitis caused by heat damage, damage to the pancreatic ducts and structures around the pancreas, and technical difficulties. EUS-RFA for the pancreas using an animal model was first reported by
Goldberg et al. ${ }^{16}$ in 1999 . They observed coagulation necrosis of approximately $10 \mathrm{~mm}$ in diameter as a result of maintaining the electrode temperature at $90 \pm 2^{\circ} \mathrm{C}$ for 6 min after inserting a $19 \mathrm{G}$ needle electrode into the tail of a pig's pancreas under EUS guidance. In early animal experimental models, there were technical difficulties, such as the location of the pancreas in the retroperitoneum, proximity to the bile duct, and distribution of main blood vessels around it. It was also difficult to set the ideal temperature for optimal thermal ablation with minimal complications. Kim et al. ${ }^{18}$ demonstrated the stability, feasibility, and efficacy of EUS-RFA on the body and tail of the pancreas in a study of 10 adult mini pigs. In this study, EUSRFA was performed on the normal pancreas of a pig for $5 \mathrm{~min}$ at a power of $50 \mathrm{~W}$ using an $18 \mathrm{G}$ needle electrode, equipped with a cooling system using cold saline inside the electrode. After RFA, coagulation necrosis with an average diameter of $23 \mathrm{~mm}$ was noted. The autopsy did not reveal any complications such as pancreatic tissue necrosis or pancreatic duct or blood vessel damage outside the RFA site. Therefore, EUSRFA for the pancreas was considered a safe, feasible, and effective treatment method. Subsequent studies investigated the technical applicability and stability of EUS-RFA for unresectable pancreatic cancer and its treatment outcomes (Table 2). In 2012, Arcidiacono et al. ${ }^{19}$ performed cryothermal ablation on 22 patients with unresectable pancreatic cancer using a bipolar RFA electrode (cryotherm probe [CTP]; ERBE, Tübingen, Germany), where the inside of the electrode was cooled with carbon dioxide. An electric power of $18 \mathrm{~W}$ was used for heating, a fixed pressure of $650 \mathrm{psi}$ was used for cooling, and the average energy transfer time was $107 \mathrm{sec}(10-360 \mathrm{sec})$. There was a significant reduction in tumor volume in 16 patients. Early complications included temporary abdominal pain (three patients) and mild duodenal bleeding (one patient); late complications included jaundice (two patients), duodenal stenosis (one patient), and fluid retention around the pancreas (one patient). In 2016, Song et al. ${ }^{6}$ published a study on EUS-RFA using an $18 \mathrm{G}$ needle electrode in six patients with unresectable pancreatic cancer. Of these, four patients had pancreatic head cancer and two had pancreatic body cancer with the median tumor size $3.8 \mathrm{~cm}$ (range, 3-9 cm). Depending on the size of the tumor, RFA was performed for $10 \mathrm{~s}$ at one site with $20-50 \mathrm{~W}$ of power, and then moved to another location until the entire tumor was cauterized. An average of 1.3 RFA sessions were conducted. When contrast-enhanced EUS was performed after the procedure, there was no contrast enhancement in the RFA-treated area, but increased blood flow was observed in the surrounding tissue. Complications included mild abdominal pain in two patients. In 2018, Scopelliti et al. ${ }^{20}$ conducted EUS-RFA on 10 patients with unresectable pancreatic cancer undergoing systemic chemotherapy using an $18 \mathrm{G}$, 
Table 2. Published Reports of Endoscopic Ultrasound-Guided Radiofrequency Ablation for Unresectable Pancreatic Cancer

\begin{tabular}{|c|c|c|c|c|c|c|c|c|c|}
\hline Study & $n$ & $\begin{array}{l}\text { Indica- } \\
\text { tion }\end{array}$ & $\begin{array}{l}\text { Mean } \\
\text { size, } \\
\text { mm } \\
\text { (range) }\end{array}$ & RFA device & Thermokinetics & $\begin{array}{l}\text { RFA } \\
\text { ses- } \\
\text { sions }\end{array}$ & Outcome & Survival & Complications \\
\hline $\begin{array}{l}\text { Arcidiacono } \\
\text { et al. }(2012)^{19}\end{array}$ & 22 & LAPC & $\begin{array}{c}36 \\
(23-54)\end{array}$ & $\begin{array}{c}\text { Cryotherm } \\
\text { probe } \\
\text { (CTP; ERBE, } \\
\text { Tübingen, } \\
\text { Germany) }\end{array}$ & $\begin{array}{l}18 \mathrm{~W} \text { (heating), } \\
650 \text { psi (cooling), } \\
107 \text { (10-360 sec) }\end{array}$ & $\mathrm{N} / \mathrm{A}$ & $\begin{array}{l}\text { Significant } \\
\text { volume } \\
\text { reduction in } \\
16 \text { patients } \\
(p=0.07)\end{array}$ & $\begin{array}{c}6 \mathrm{mo} \\
(1-12)\end{array}$ & $\begin{array}{l}\text { Early: three transient ab- } \\
\text { dominal pain, one minor } \\
\text { duodenal bleeding } \\
\text { Late: two jaundice, one } \\
\text { duodenal stricture, one } \\
\text { cystic fluid collection }\end{array}$ \\
\hline $\begin{array}{l}\text { Song et al. } \\
(2016)^{6}\end{array}$ & 6 & $\begin{array}{l}\text { LAPC (4), } \\
\text { metastatic } \\
\text { PC (2) }\end{array}$ & $\begin{array}{c}38 \\
(30-90)\end{array}$ & $\begin{array}{l}18 \text { G RFA } \\
\text { electrode } \\
\text { (EUSRA; } \\
\text { STARmed, } \\
\text { Goyang, } \\
\text { Korea) }\end{array}$ & $20-50 \mathrm{~W}, 10 \mathrm{sec}$ & $\begin{array}{c}1.3 \\
(1-2)\end{array}$ & $\begin{array}{l}\text { Necrosis at } \\
\text { ablation site } \\
\text { on CE-EUS }\end{array}$ & N/A & Two mild abdominal pain \\
\hline $\begin{array}{l}\text { Scopelliti et } \\
\text { al. }(2018)^{20}\end{array}$ & 10 & LAPC & $\begin{array}{c}45 \\
(25-70)\end{array}$ & $\begin{array}{l}18 \text { G RFA } \\
\text { electrode } \\
\text { (EUSRA; } \\
\text { STARmed) }\end{array}$ & $\begin{array}{l}20-30 \mathrm{~W}, 285 \\
(100-560 \mathrm{sec})\end{array}$ & $\begin{array}{c}1.4 \\
(1-2)\end{array}$ & $\begin{array}{l}\text { Hypodense } \\
\text { ablated area } \\
\text { on CT scan } \\
\text { at 30-day }\end{array}$ & N/A & $\begin{array}{l}\text { Two asymptomatic ascites, } \\
\text { two asymptomatic peri- } \\
\text { pancreatic effusion }\end{array}$ \\
\hline
\end{tabular}

CE-EUS, contrast-enhanced endoscopic ultrasound; CT, computed tomography; LAPC, locally advanced pancreatic cancer; N/A, not available; PC, pancreatic cancer; RFA, radiofrequency ablation.

$10 \mathrm{~mm}$ needle electrode. They used $20-30 \mathrm{~W}$ of power, and the average energy transfer time was $285 \mathrm{sec}(100-560 \mathrm{sec})$. Abdominal computed tomography (CT) performed 30 days after the procedure to evaluate treatment response showed low-density necrotic areas inside the tumor in all 10 patients. Procedural complications included mild asymptomatic ascites (two patients) and fluid retention around the pancreas (two patients), but there were no serious complications associated with the procedure. The results of the above animal and human experiments indicate that EUS-RFA is technically safe for patients with unresectable pancreatic cancer. It may be used as an aid to existing systemic chemotherapy or considered as an alternative treatment for patients at high risk of systemic adverse events of chemotherapy.

\section{Irreversible electroporation}

IRE is a non-thermal resection technique that locally resects a tumor using high-voltage electric pulses generated by needle electrodes inserted into and around the tumor. Electric pulses induce irreversible damage by producing nanopores in the tumor cell membrane to induce apoptosis. ${ }^{21}$ Martin et al. ${ }^{22}$ published a study conducted on patients with unresectable pancreatic cancer, where the prognosis of a group treated with a combination of standard therapy (chemotherapy \pm radiotherapy) and IRE (54 patients) was compared to that of a group treated with standard therapy alone (85 patients). There was a significant improvement in the patient group treated with IRE in terms of local progression-free survival (14 months vs. 6 months, $p=0.01$ ), distant progression-free survival (15 months vs. 9 months, $p=0.02$ ), and overall survival (20 months vs. 13 months, $p=0.03$ ). IRE has been reported to minimize thermal damage, unlike RFA, which can cause thermal damage to major blood vessels, bile ducts, and nerves around the pancreas. However, in some conditions using high-intensity electric currents, IRE has also been reported to cause coagulation necrosis, similar to RFA. This effect is likely due to the partial thermal damage caused by high-intensity currents. ${ }^{23}$ Therefore, further study on the stability of IRE and development of tools to safely apply this technique is required. Conventional IRE is performed using a CT-guided percutaneous method by inserting electrodes into the tumor during open or laparoscopic surgery. However, the development of EUS technology and equipment allows IRE to be performed more safely and accurately under EUS guidance. Recently, Lee et al. ${ }^{24}$ published a study on the effectiveness and reproducibility of EUS-guided IRE using animal experiments, with new electrodes developed for use in EUS. Further studies on the development of a standardized needle electrode that can be used for EUS, identification of safe and effective voltage settings, and demonstration of clinical applications are required. 


\section{RADIOTHERAPY}

\section{Endoscopic ultrasound-guided fiducial marker insertion}

Stereotactic body radiation therapy (SBRT) is a method of delivering intensively high doses of radiation to a tumor site using a cyberknife or a similar tool. The treatment period is short when compared to that of conventional radiotherapy, and several studies have proven it to be effective even against unresectable pancreatic cancer. Jung et $\mathrm{al}^{25}$ conducted a retrospective analysis of the treatment outcomes of 95 patients with unresectable pancreatic cancer treated in a single institution between 2011 and 2016. They reported that freedom from local disease progression and overall survival were better, with lower treatment toxicity, in patients who simultaneously received systemic chemotherapy and SBRT. A significant challenge in applying SBRT to pancreatic cancer is the necessity to navigate movement caused by breathing. Unlike intracranial tumors, the pancreas does not exist in a fixed space such as the skull, and is estimated to move $2-3 \mathrm{~cm}$ during the breathing cycle. The fiducial marker inserted into the border area of pancreatic cancer acts as a fixed reference point in the tumor, helping in accounting the movement of the target during stereotactic radiotherapy. Traditionally, fiducial markers were inserted percutaneously under CT or ultrasound guidance. However, the location of the pancreas in the retroperitoneal space makes percutaneous fiducial marker insertion risky, causing potential vascular damage due to poor accuracy. Park et al. ${ }^{26}$ predicted that EUS-guided insertion of fiducial markers using real-time Doppler imaging could decrease the risk of bleeding. They performed EUS-guided fiducial marker insertion in 57 patients with unresectable pancreatic cancer, and minor bleeding was reported in only one patient.

Although rare, another risk of the percutaneous approach is peritoneal dissemination of the tumor. During CT or ultrasound-guided fine needle aspiration, peritoneal dissemination of the tumor occurs in $0.005 \%-0.009 \%$ of all cases. However, the EUS-guided approach is thought to have a lower risk of peritoneal dissemination because the perforation pathway is very short compared to percutaneous methods. ${ }^{27}$

Thus, EUS-guided fiducial marker insertion is rapidly becoming an essential procedure when preparing for SBRT in patients with unresectable pancreatic cancer. It is a technically safe and accurate approach compared to other mesthods.

\section{Endoscopic ultrasound-guided brachytherapy}

Brachytherapy is a method of treating tumors by inserting a radioactive source into or around the tumor tissue. Radioactive sources used for brachytherapy include iodine- 125 , iridi- um-192, palladium-103, and iodine-125, the last of which has the longest half-life and is thought to be useful for pancreatic cancer with rapid tumor growth. Bhutani et al. ${ }^{28}$ reported a $58 \%$ reduction in tumor volume in patients with unresectable pancreatic cancer treated with brachytherapy using a new radioactive source, phosphorus-32 microparticles (P-32; OncoSil Medical Ltd., Sydney, Australia), in combination with standard chemotherapy. The development of new radioactive sources and more data on treatment outcomes are required in the future.

\section{CONCLUSIONS}

Recent improvements in EUS equipment and proficiency of clinicians have expanded the type and scope of endoscopic local treatment at par with the conventional treatment of pancreatic cancer. Endoscopic local treatment for unresectable pancreatic cancer may reduce the size of the tumor, making surgical resection possible. Ultimately, it is expected to improve the survival rate of patients with advanced pancreatic cancer, and simultaneously control complications such as pain and biliary obstruction, thereby improving the quality of life. To achieve this goal, continuous development of endoscopic equipment and various accessory devices and multidisciplinary clinical research should be actively pursued.

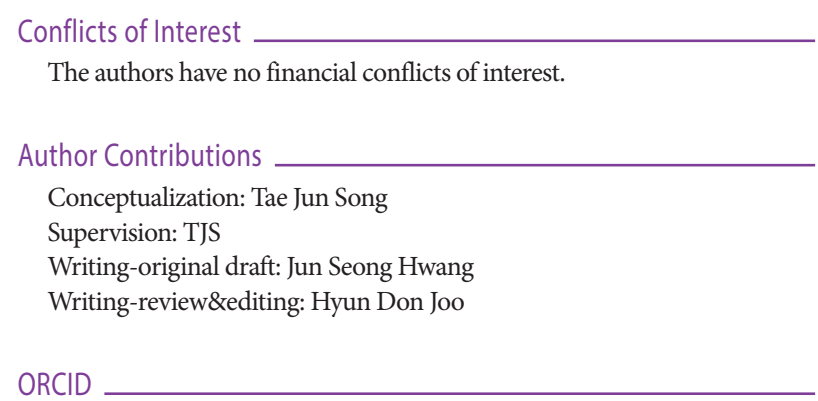

Jun Seong Hwang: https://orcid.org/0000-0002-0454-7527

Hyun Don Joo: https://orcid.org/0000-0001-9477-1380

\section{REFERENCES}

1. National Cancer Center. Cancer statistics for 2016 [Internet]. Goyang: National Cancer Center; c2019 [updated 2019 Jan 4]. Available from: https://ncc.re.kr/cancerStatsView.ncc?bbsnum=459\&searchKey=total\&searchValue=\&pageNum=1,\%202019.

2. Ducreux M, Cuhna AS, Caramella C, et al. Cancer of the pancreas: ESMO clinical practice guidelines for diagnosis, treatment and follow-up. Ann Oncol 2015;26(Suppl 5):v56-v68.

3. Gurusamy KS, Kumar S, Davidson BR, Fusai G. Resection versus other treatments for locally advanced pancreatic cancer. Cochrane Database Syst Rev 2014;(2):CD010244. 
4. Stark A, Donahue TR, Reber HA, Hines OJ. Pancreatic cyst disease: a review. JAMA 2016;315:1882-1893.

5. Böttger TC, Junginger T. Factors influencing morbidity and mortality after pancreaticoduodenectomy: critical analysis of 221 resections. World J Surg 1999;23:164-171; discussion 171-172.

6. Song TJ, Seo DW, Lakhtakia S, et al. Initial experience of EUS-guided radiofrequency ablation of unresectable pancreatic cancer. Gastrointest Endosc 2016;83:440-443.

7. Feig C, Gopinathan A, Neesse A, Chan DS, Cook N, Tuveson DA. The pancreas cancer microenvironment. Clin Cancer Res 2012;18:42664276.

8. Levy MJ, Alberts SR, Chari ST, et al. EUS guided intra-tumoral gemcitabine therapy for locally advanced and metastatic pancreatic cancer Gastrointest Endosc 2011;73(4 Suppl):AB144-AB145

9. Matthes K, Mino-Kenudson M, Sahani DV, et al. EUS-guided injection of paclitaxel (OncoGel) provides therapeutic drug concentrations in the porcine pancreas (with video). Gastrointest Endosc 2007;65:448-453

10. Vacchelli E, Eggermont A, Sautès-Fridman C, et al. Trial watch: oncolytic viruses for cancer therapy. Oncoimmunology 2013;2:e24612.

11. Hecht JR, Bedford R, Abbruzzese JL, et al. A phase I/II trial of intratumoral endoscopic ultrasound injection of ONYX-015 with intravenous gemcitabine in unresectable pancreatic carcinoma. Clin Cancer Res 2003;9:555-561.

12. Nakao A, Kasuya H, Sahin TT, et al. A phase I dose-escalation clinical trial of intraoperative direct intratumoral injection of HF10 oncolytic virus in non-resectable patients with advanced pancreatic cancer. Cancer Gene Ther 2011;18:167-175.

13. Chang KJ, Nguyen PT, Thompson JA, et al. Phase I clinical trial of allogeneic mixed lymphocyte culture (cytoimplant) delivered by endoscopic ultrasound-guided fine-needle injection in patients with advanced pancreatic carcinoma. Cancer 2000;88:1325-1335.

14. Nonogaki K, Hirooka Y, Itoh A, et al. Combined treatment with immunotherapy and chemotherapy using endoscopic ultrasonography: a phase 1 trial as first-line treatment in patients with locally advanced pancreatic carcinoma. Gastrointest Endosc 2007;65:AB207.

15. Hecht JR, Farrell JJ, Senzer N, et al. EUS or percutaneously guided intratumoral TNFerade biologic with 5-fluorouracil and radiotherapy for first-line treatment of locally advanced pancreatic cancer: a phase I/II study. Gastrointest Endosc 2012;75:332-338.
16. Goldberg SN, Mallery S, Gazelle GS, Brugge WR. EUS-guided radiofrequency ablation in the pancreas: results in a porcine model. Gastrointest Endosc 1999;50:392-401.

17. Teng LS, Jin KT, Han N, Cao J. Radiofrequency ablation, heat shock protein 70 and potential anti-tumor immunity in hepatic and pancreatic cancers: a minireview. Hepatobiliary Pancreat Dis Int 2010;9:361-365.

18. Kim HJ, Seo DW, Hassanuddin A, et al. EUS-guided radiofrequency ablation of the porcine pancreas. Gastrointest Endosc 2012;76:1039-1043.

19. Arcidiacono PG, Carrara S, Reni M, et al. Feasibility and safety of EUS-guided cryothermal ablation in patients with locally advanced pancreatic cancer. Gastrointest Endosc 2012;76:1142-1151.

20. Scopelliti F, Pea A, Conigliaro R, et al. Technique, safety, and feasibility of EUS-guided radiofrequency ablation in unresectable pancreatic cancer. Surg Endosc 2018;32:4022-4028.

21. Rubinsky B, Onik G, Mikus P. Irreversible electroporation: a new ablation modality--clinical implications. Technol Cancer Res Treat 2007;6:37-48.

22. Martin RC 2nd, McFarland K, Ellis S, Velanovich V. Irreversible electroporation in locally advanced pancreatic cancer: potential improved overall survival. Ann Surg Oncol 2013;20(Suppl 3):S443-S449.

23. Faroja M, Ahmed M, Appelbaum L, et al. Irreversible electroporation ablation: is all the damage nonthermal? Radiology 2013;266:462-470.

24. Lee JM, Choi HS, Chun HJ, et al. EUS-guided irreversible electroporation using endoscopic needle-electrode in porcine pancreas. Surg Endosc 2019;33:658-662.

25. Jung J, Yoon SM, Park JH, et al. Stereotactic body radiation therapy for locally advanced pancreatic cancer. PLoS One 2019;14:e0214970.

26. Park WG, Yan BM, Schellenberg D, et al. EUS-guided gold fiducial insertion for image-guided radiation therapy of pancreatic cancer: 50 successful cases without fluoroscopy. Gastrointest Endosc 2010;71:513518.

27. Choi JH, Seo DW, Park DH, Lee SK, Kim MH. Fiducial placement for stereotactic body radiation therapy under only endoscopic ultrasonography guidance in pancreatic and hepatic malignancy: practical feasibility and safety. Gut Liver 2014;8:88-93.

28. Bhutani MS, Cazacu IM, Luzuriaga Chavez AA, et al. Novel EUS-guided brachytherapy treatment of pancreatic cancer with phosphorus-32 microparticles: first United States experience. VideoGIE 2019;4:223-225. 